Vietnam Journal of Mechanics, VAST, Vol.38, No. 1 (2016), pp. 27 -38

DOI:10.15625/0866-7136/38/1/6191

\title{
THE DISPERSION OF RAYLEIGH WAVES IN ORTHOTROPIC LAYERED HALF-SPACE USING MATRIX METHOD
}

\author{
Tran Thanh Tuan ${ }^{1, *}$, Tran Ngoc Trung ${ }^{2}$ \\ ${ }^{1}$ VNU University of Science, Hanoi, Vietnam \\ ${ }^{2}$ Publishing House for Science and Technology, VAST, Hanoi, Vietnam \\ *E-mail: tranthanhtuan@hus.edu.vn \\ Received May 14, 2015
}

\begin{abstract}
In this paper, the secular equation of Rayleigh surface waves propagating in an orthotropic layered half-space is derived by the matrix method. All the layers and the halfspace are assumed to have identical principle axes. The explicit form of the matrizant for each layer is obtained by the Sylvester's theorem. The derived secular equation takes only real values and depends only on the dimensionless variables and dimensionless material parameters. Hence, it is convenient in numerical calculation.
\end{abstract}

Keywords: Rayleigh waves, matrix method, Sylvester's theorem, orthotropy.

\section{INTRODUCTION}

The study of Rayleigh surface waves propagation in layered half-space is of considerable interest in the field of seismology since the Earth's surface could be considered to consist of several layers overlying a half-space. The first systematic and efficient method to find the secular equation is the propagator matrix method proposed by Thomson [1] and Haskell [2]. This method expresses the relation between the displacements and the stresses at two faces of a layer by a matrix called the layer propagator matrix. The product of these matrices is used to find relation of the displacements and stresses at the free surface and at the top of the half-space to formulate the secular equation using boundary conditions. This method has been modified to be more efficient and stable such as in Knopoff [3], Dunkin [4], Kennett [5] and Chen [6] and has been used widely recently.

For the homogeneous isotropic layered half-space, the layer propagator matrix has an explicit form because the vertical wave numbers in the layer are simple and always either real or pure imaginary. The situation become more complicated for the homogeneous anisotropic layers in which the vertical wave number could be complex. This make the propagator matrix could be complex leading to difficulties in numerical calculation and it is also not easy to have the layer propagator matrix in explicit form (see Crampin [7], Crampin and Taylor [8]). This also makes difficulties in handling the decay

(c) 2016 Vietnam Academy of Science and Technology 
condition in the half-space. For the orthotropic layers, an explicit expression of the transfer matrix is given in Solyanik [9] and its more convenient in numerical calculation form is then given in Rokhlin and Wang [10]. However, the elements of these transfer matrices are not always real.

In this paper, a more advanced formulation of the Thomson-Haskell method is used to obtain the matrizant of a layer, which is analogous to the layer propagator matrix, first. It is written in explicit form by the use of Sylvester's theorem. For the purpose of finding the secular equation, an equivalent dimensionless and real form of the matrizant is derived. Then, the difficulty in the decay condition will be overcome by using the Vieta's formulas for the propagation equation of the half-space. Finally, the secular equation in convenient form for numerical calculation is obtained and an numerical example in finding the dispersive curves of Rayleigh surface waves is presented.

\section{FORMULATION OF THE PROBLEM}

Consider a stack of $n$ homogeneous orthotropic layers overlying on a homogeneous orthotropic half-space. By $h^{(k)}, \rho^{(k)}, c_{i j}^{(k)}(i, j=\overline{1,6})$ we denote the thickness, the density, and the material constants of the $k$ th layer $(k=\overline{0, n})$. The underlying half-space is labeled as the zeroth layer $(k=0)$. All the layers are assumed to have identical principal axes and the waves propagates along $x_{1}$-axis which is one of the three principal axes (see Fig. 1).

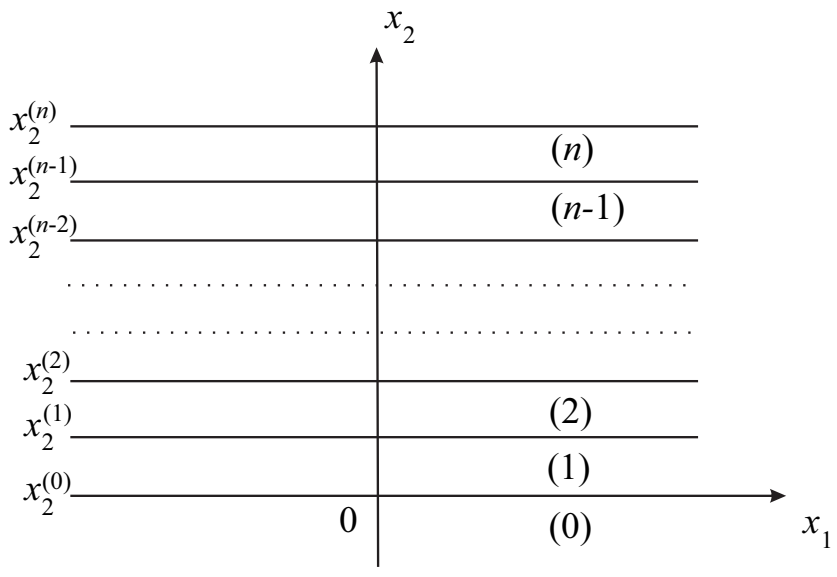

Fig. 1. Model of $n$ orthotropic layers overlying an orthotropic half-space.

The half-space is labeled as layer (0)

Consider plane waves propagating in a layer whose material parameters are denoted by $\left(\rho, c_{i j}\right)$ for the moment such that

$$
\begin{aligned}
& u_{1}=-i y_{2}\left(x_{2} ; \omega, k\right) e^{i\left(\omega t-k x_{1}\right)}, \\
& u_{2}=y_{1}\left(x_{2} ; \omega, k\right) e^{i\left(\omega t-k x_{1}\right)}, \\
& u_{3}=0,
\end{aligned}
$$


where $\omega, k, t$ are the angular frequency, horizontal wave number and the time, respectively.

Since the layer is made of orthotropic elastic material, then the strain-stress relations are (see Ting [11])

$$
\begin{aligned}
& \sigma_{11}=c_{11} u_{1,1}+c_{12} u_{2,2}, \\
& \sigma_{22}=c_{12} u_{1,1}+c_{22} u_{2,2}, \\
& \sigma_{12}=c_{66}\left(u_{1,2}+u_{2,1}\right),
\end{aligned}
$$

where $\sigma_{i j}$ are the stresses of the layer, commas indicate differentiation with respect to spatial variable $x_{k}$. In the absence of body forces, equations of motion are

$$
\begin{aligned}
& \sigma_{11,1}+\sigma_{12,2}=\rho \ddot{u}_{1}, \\
& \sigma_{12,1}+\sigma_{22,2}=\rho \ddot{u}_{2} .
\end{aligned}
$$

Let

$$
\begin{aligned}
& \sigma_{22}=y_{3} e^{i\left(\omega t-k x_{1}\right)}, \\
& \sigma_{12}=-i y_{4} e^{i\left(\omega t-k x_{1}\right)},
\end{aligned}
$$

then substituting (1) into (2) we obtain

$$
\begin{aligned}
& y_{3}=c_{22} \frac{d y_{1}}{d x_{2}}-k c_{12} y_{2}, \\
& y_{4}=c_{66}\left(\frac{d y_{1}}{d x_{2}}+k y_{1}\right),
\end{aligned}
$$

and

$$
\begin{aligned}
& k\left(c_{12} \frac{d y_{1}}{d x_{2}}-k c_{11} y_{2}\right)+\frac{d y_{4}}{d x_{2}}=-\rho \omega^{2} y_{2} \\
& -k y_{4}+\frac{d y_{3}}{d x_{2}}=-\rho \omega^{2} y_{1} .
\end{aligned}
$$

Eqs. (5) and (6) can be written in the matrix form as follows

$$
\frac{d \mathbf{y}}{d x_{2}}=\mathbf{U y},
$$

where $\mathbf{y}=\left[y_{1}, y_{2}, y_{3}, y_{4}\right]^{T}$ (the symbol " $T$ " indicates the transpose) and

$$
\mathbf{U}=\left[\begin{array}{cccc}
0 & k \frac{c_{12}}{c_{22}} & \frac{1}{c_{22}} & 0 \\
-k & 0 & 0 & \frac{1}{c_{66}} \\
-\rho \omega^{2} & 0 & 0 & k \\
0 & k^{2}\left(c_{11}-\frac{c_{12}^{2}}{c_{22}}\right)-\rho \omega^{2} & -k \frac{c_{12}}{c_{22}} & 0
\end{array}\right] .
$$

The propagation equation of the layer, which is the characteristic equation determining the eigenvalues $\lambda=k b$ of the matrix $\mathbf{U}$, is

$$
c_{22} c_{66} b^{4}+\left[\left(c_{12}+c_{66}\right)^{2}+c_{22}\left(X-c_{11}\right)+c_{66}\left(X-c_{66}\right)\right] b^{2}+\left(c_{11}-X\right)\left(c_{66}-X\right)=0,
$$


where $X=\rho c^{2}$. This is a quadratic equation in $b^{2}$ with solution $b_{1}^{2}$ and $b_{2}^{2}$ satisfying Vieta's formulas

$$
\begin{aligned}
& b_{1}^{2}+b_{2}^{2}=-\frac{\left(c_{12}+c_{66}\right)^{2}+c_{22}\left(X-c_{11}\right)+c_{66}\left(X-c_{66}\right)}{c_{22} c_{66}}=S \\
& b_{1}^{2} \cdot b_{2}^{2}=\frac{\left(c_{11}-X\right)\left(c_{66}-X\right)}{c_{22} c_{66}}=P .
\end{aligned}
$$

Eq. (9) has four roots, namely $\pm \sqrt{b_{1}^{2}}$ and $\pm \sqrt{b_{2}^{2}}$. When $S^{2}-4 P>0$ the four roots are either real or pure imaginary. Otherwise, they are complex conjugate in pairs and note that

$$
b_{1}^{2}=\left(b_{2}^{2}\right)^{*}
$$

in which the asterisk denotes the complex conjugate. The eigenvector of matrix $\mathbf{U}$ corresponding to eigenvalue $\lambda=k b$ is

$$
\mathbf{v}=\left[\begin{array}{c}
b\left(c_{12}+c_{66}\right) \\
b^{2} c_{22}-c_{66}+X \\
-k\left[c_{12} X-\left(b^{2} c_{22}+c_{12}\right) c_{66}\right] \\
k c_{66} b\left(b^{2} c_{22}+c_{12}+X\right)
\end{array}\right] .
$$

Consider the Rayleigh surface waves propagating in layered half-space (Fig. 1) along the $x_{1}$-axis. The governing equations of the motion of Rayleigh waves in this model are

$$
\frac{d \mathbf{y}}{d x_{2}}=\mathbf{U}\left(x_{2}\right) \mathbf{y}
$$

in which the coefficient matrix is a function of $x_{2}$ defined as

$$
\begin{aligned}
& \mathbf{U}\left(x_{2}\right)=\mathbf{U}^{(i)}, \quad\left(x_{2} \in\left[x_{2}^{(i-1)}, x_{2}^{(i)}\right], i=\overline{1, n}\right), \\
& \mathbf{U}\left(x_{2}\right)=\mathbf{U}^{(0)}, \quad\left(x_{2} \in[-\infty, 0]\right),
\end{aligned}
$$

where $\mathbf{U}^{(i)}$ is of form (8) with all material parameters are replaced by those of $i^{\text {th }}$ layer.

The boundary conditions are the traction free at the top surface, i.e.

$$
y_{3}(H ; \omega, k)=y_{4}(H ; \omega, k)=0,
$$

where $H=\sum h_{i}$ is the total thickness of the layers, and the continuity of the displacements and stresses at each interface, i.e.

$$
\mathbf{y}^{(i)}\left(x_{2}^{(i)} ; \omega, k\right)=\mathbf{y}^{(i+1)}\left(x_{2}^{(i)} ; \omega, k\right),(i=\overline{0, n-1})
$$

where $\mathbf{y}^{(i)}\left(x_{2} ; \omega, k\right)=\mathbf{y}\left(x_{2} ; \omega, k\right)$ at $x_{2}=\left[x_{2}^{(i-1)}, x_{2}^{(i)}\right],(i=\overline{1, n})$ and the decay condition that

$$
\mathbf{y}\left(x_{2} ; \omega, k\right) \rightarrow 0 \quad \text { as } \quad x_{2} \rightarrow-\infty
$$

The differential equations (13) along with the boundary conditions (15), (16) and (17) will be used to determine the secular equation of the Rayleigh surface waves. 


\section{THE MATRIX METHOD}

Consider the differential equations (13). A formal solution to this equation is

$$
\mathbf{y}\left(x_{2}\right)=\mathbf{\Omega}\left(x_{2}, x_{2}^{\prime}\right) \mathbf{y}\left(x_{2}^{\prime}\right),
$$

where $\mathbf{y}\left(x_{2}^{\prime}\right)$ is the given initial value of $\mathbf{y}$ at $x_{2}=x_{2}^{\prime}$ and $\boldsymbol{\Omega}\left(x_{2}, x_{2}^{\prime}\right)$ is called the matrizant of $\mathbf{U}$. When $\mathbf{U}$ is a constant matrix, the matrizant is (see Frazer et. al [12])

$$
\boldsymbol{\Omega}\left(x_{2}, x_{2}^{\prime}\right)=\exp \left[\left(x_{2}-x_{2}^{\prime}\right) \mathbf{U}\right] \text {. }
$$

Let $n$ be the rank of $\mathbf{U}$ and $\lambda_{i}(i=1,2, \ldots, n)$ be the eigenvalues of $\mathbf{U}$, by Sylvester's theorem (e.g. see Frazer et. al [12]), we have

$$
\mathbf{E}(h)=\exp (h \mathbf{U})=\sum_{i=1}^{n} e^{\lambda_{i} h} \prod_{j \neq i} \frac{\lambda_{j} \mathbf{I}-\mathbf{U}}{\lambda_{j}-\lambda_{i}} .
$$

Applying this result to our layered model, for example to the $1^{\text {st }}$ layer, we have

$$
\mathbf{y}\left(x_{2}^{(1)}\right)=\mathbf{E}^{(1)}\left(h_{1}\right) \mathbf{y}\left(x_{2}^{(0)}\right)=\mathbf{E}^{(1)}\left(h_{1}\right) \mathbf{y}(0),
$$

where $\mathbf{E}^{(1)}\left(h_{1}\right)=\exp \left(h_{1} \mathbf{U}^{(1)}\right)$ computed from (20) for matrix $\mathbf{U}^{(1)}$. Using this procedure for other layers, we have

$$
\begin{aligned}
\mathbf{y}(H)=\mathbf{y}\left(x_{2}^{(n)}\right) & =\mathbf{E}^{(n)}\left(h_{n}\right) \mathbf{y}\left(x_{2}^{(n-1)}\right) \\
& =\mathbf{E}^{(n)}\left(h_{n}\right) \mathbf{E}^{(n-1)}\left(h_{n-1}\right) \mathbf{y}\left(x_{2}^{(n-2)}\right)=\cdots= \\
& =\mathbf{E}^{(n)}\left(h_{n}\right) \mathbf{E}^{(n-1)}\left(h_{n-1}\right) \ldots \mathbf{E}^{(2)}\left(h_{2}\right) \mathbf{E}^{(1)}\left(h_{1}\right) \mathbf{y}(0),
\end{aligned}
$$

where $\mathbf{E}^{(i)}\left(h_{i}\right)=\exp \left(h_{i} \mathbf{U}^{(i)}\right),(i=\overline{1, n})$.

To obtain $\mathbf{y}(0)$, we consider the system of waves propagating in the half-space. The wave system in the half-space is controlled by Eq. (7) with $\mathbf{U}$ replaced by $\mathbf{U}^{(0)}$. In general, Eq. (7) has four fundamental solutions of form

$$
\mathbf{y}_{j}^{(0)}\left(x_{2} ; \omega, k\right)=\mathbf{v}_{j}^{(0)} \exp \left(\lambda_{j}^{(0)} x_{2}\right), \quad(j=\overline{1,4})
$$

where $\lambda_{j}^{(0)}$ are the eigenvalues of matrix $\mathbf{U}^{(0)}$ determined from (9) and $\mathbf{v}_{j}^{(0)}$ are the corresponding eigenvectors computed from (12). For the sake of simplicity, hereafter we denote $\mathbf{y}^{(i)}\left(x_{2} ; \omega, k\right)$ by $\mathbf{y}^{(i)}\left(x_{2}\right)$.

Due to the decay condition, there are only two solutions in the half-space with the eigenvalue having positive real part. These two eigenvalues are $k b_{1}^{(0)}, k b_{2}^{(0)}$ and determined from (9). Hence, the displacement field in the half-space is

$$
\mathbf{y}^{(0)}\left(x_{2}\right)=C_{1} \mathbf{v}_{1}^{(0)} e^{k b_{1}^{(0)} x_{2}}+C_{2} \mathbf{v}_{2}^{(0)} e^{k b_{2}^{(0)} x_{2}},
$$

where $C_{1}$ and $C_{2}$ are constant. At $x_{2}=0$, we have

$$
\mathbf{y}(0)=\mathbf{y}^{(0)}(0)=C_{1} \mathbf{v}_{1}^{(0)}+C_{2} \mathbf{v}_{2}^{(0)} \text {. }
$$


Denote

$$
\mathbf{E}(H)=\mathbf{E}^{(n)}\left(h_{n}\right) \mathbf{E}^{(n-1)}\left(h_{n-1}\right) \ldots \mathbf{E}^{(2)}\left(h_{2}\right) \mathbf{E}^{(1)}\left(h_{1}\right),
$$

we have from Eq. (22) that

$$
\mathbf{y}(H)=C_{1} \mathbf{E}(H) \mathbf{v}_{1}^{(0)}+C_{3} \mathbf{E}(H) \mathbf{v}_{2}^{(0)} .
$$

The traction free condition (15) gives

$$
\begin{aligned}
& y_{3}(H)=0 \\
& y_{4}(H)=0 .
\end{aligned}
$$

These are two homogeneous equations of $C_{1}$ and $C_{2}$. Hence, to obtain the nontrivial solution, we must have

$$
\Delta(\omega, k)=\left[E_{3 i}(H) \mathbf{v}_{1}^{(0)}(i)\right]\left[E_{4 i}(H) \mathbf{v}_{2}^{(0)}(i)\right]-\left[E_{3 i}(H) \mathbf{v}_{2}^{(0)}(i)\right]\left[E_{4 i}(H) \mathbf{v}_{1}^{(0)}(i)\right]=0,
$$

in which the Einstein summation for $i$ from 1 to 4 is applied. This is the secular equation of Rayleigh waves and it could be used to obtain the dispersion curves numerically such as by some numerical integral method (e.g see Takeuchi and Saito [13]). The numerical approach using Eq. (29) has some intrinsic difficulties but a problem could be arisen here is how to choose two out of four solutions in the half-space satisfying the decay condition because the eigenvalues of the propagation equation of the half-space are in general complex. In the following, the secular equation will be written in a new form to avoid the above difficulties.

Eq. (29) could be expressed as

$$
\Delta(\omega, k)=\mathbf{M} \cdot \mathbf{V}=0,
$$

where $\mathbf{M}$ and $\mathbf{V}$ are two vectors of six components defined as

$$
\mathbf{V}=\left[\begin{array}{ccc}
\mathbf{v}_{1}^{(0)}(1) \mathbf{v}_{2}^{(0)}(2)-\mathbf{v}_{2}^{(0)}(1) \mathbf{v}_{1}^{(0)}(2) \\
\mathbf{v}_{1}^{(0)}(1) \mathbf{v}_{2}^{(0)}(3)-\mathbf{v}_{2}^{(0)}(1) \mathbf{v}_{1}^{(0)}(3) \\
\mathbf{v}_{1}^{(0)}(1) \mathbf{v}_{2}^{(0)}(4)-\mathbf{v}_{2}^{(0)}(1) \mathbf{v}_{1}^{(0)}(4) \\
\mathbf{v}_{1}^{(0)}(2) \mathbf{v}_{2}^{(0)}(3)-\mathbf{v}_{2}^{(0)}(2) \mathbf{v}_{1}^{(0)}(3) \\
\mathbf{v}_{1}^{(0)}(2) \mathbf{v}_{2}^{(0)}(4)-\mathbf{v}_{2}^{(0)}(2) \mathbf{v}_{1}^{(0)}(4) \\
\mathbf{v}_{1}^{(0)}(3) \mathbf{v}_{2}^{(0)}(4)-\mathbf{v}_{2}^{(0)}(3) \mathbf{v}_{1}^{(0)}(4)
\end{array}\right] \text { and } \mathbf{M}=\left[\begin{array}{l}
E_{31}(H) E_{42}(H)-E_{41}(H) E_{32}(H) \\
E_{31}(H) E_{43}(H)-E_{41}(H) E_{33}(H) \\
E_{31}(H) E_{44}(H)-E_{41}(H) E_{34}(H) \\
E_{32}(H) E_{43}(H)-E_{42}(H) E_{33}(H) \\
E_{32}(H) E_{44}(H)-E_{42}(H) E_{34}(H) \\
E_{33}(H) E_{44}(H)-E_{43}(H) E_{34}(H)
\end{array}\right]
$$

Note that $b_{1}^{(0)}=\left(b_{2}^{(0)}\right)^{*}$. Therefore, $\mathbf{v}_{1}^{(0)}=\left(\mathbf{v}_{2}^{(0)}\right)^{*}$ and we can write vector $\mathbf{V}$ as

$$
\mathbf{V}=\left(b_{1}^{(0)}-b_{2}^{(0)}\right) k\left(c_{66}^{(0)}\right)^{3}\left[\frac{\bar{V}_{1}}{k c_{66}^{(0)}}, \bar{V}_{2}, \bar{V}_{3}, \bar{V}_{4}, \bar{V}_{5}, k c_{66}^{(0)} \bar{V}_{6}\right]^{T},
$$


where $\mathbf{\mathbf { V }}=\left[\bar{V}_{1}, \bar{V}_{2}, \bar{V}_{3}, \bar{V}_{4}, \bar{V}_{5}, \bar{V}_{6}\right]^{T}$ is a dimensionless vector whose components are

$$
\begin{aligned}
\bar{V}_{1}= & \left(e_{3}^{(0)}+1\right)\left[-e_{2}^{(0)} \sqrt{P^{(0)}}-1+x^{(0)}\right] \\
\bar{V}_{2}= & -\left(e_{3}^{(0)}+1\right)\left(e_{3}^{(0)} x^{(0)}+e_{2}^{(0)} \sqrt{P^{(0)}}-e_{3}^{(0)}\right) \\
\bar{V}_{3}= & -\left(e_{3}^{(0)}+1\right) e_{2}^{(0)} \sqrt{P^{(0)}} \sqrt{S^{(0)}+2 \sqrt{P^{(0)}}} \\
\bar{V}_{4}= & -\left(e_{3}^{(0)}+1\right) e_{2}^{(0)}\left(x^{(0)}-1\right) \sqrt{S^{(0)}+2 \sqrt{P^{(0)}}}, \\
\bar{V}_{5}= & -\left(e_{2}^{(0)}\right)^{2} P^{(0)}+e_{2}^{(0)}\left(1-x^{(0)}\right) S^{(0)}+e_{2}^{(0)}\left(e_{3}^{(0)}+1\right) \sqrt{P^{(0)}}+ \\
& +\left(e_{3}^{(0)}+x^{(0)}\right)\left(1-x^{(0)}\right) \\
\bar{V}_{6}= & e_{2}^{(0)} e_{3}^{(0)}\left(x^{(0)}-1\right) S^{(0)}-\left(e_{2}^{(0)}\right)^{2} P^{(0)}+e_{2}^{(0)}\left(e_{3}^{(0)}+1\right) x^{(0)} \sqrt{P^{(0)}}+ \\
& +e_{3}^{(0)}\left(e_{3}^{(0)}+x^{(0)}\right)\left(x^{(0)}-1\right)
\end{aligned}
$$

In this equation, we use the dimensionless quantities defined as

$$
e_{1}^{(i)}=\frac{c_{11}^{(i)}}{c_{66}^{(i)}}, \quad e_{2}^{(i)}=\frac{c_{22}^{(i)}}{c_{66}^{(i)}}, \quad e_{3}^{(i)}=\frac{c_{12}^{(i)}}{c_{66}^{(i)}}, \quad x^{(i)}=\frac{X^{(i)}}{c_{66}^{(i)}}, \quad(i=\overline{0, n})
$$

and $S^{(0)}$ and $P^{(0)}$ are determined from (10) with parameters of the half-space. Note that for Rayleigh waves existence in the half-space, i.e. $b_{1}^{(0)}$ and $b_{2}^{(0)}$ having real part, then (see Vinh and Ogden [14])

$$
0<X^{(0)}<\min \left\{c_{66}^{(0)}, c_{11}^{(0)}\right\}
$$

which makes $P^{(0)}$ positive.

To calculate vector $\mathbf{M}$ in Eq. (31), we need an explicit form of layer matrizant expressed in Eq. (20). The eigenvalues of matrix $\mathbf{U}^{(i)}$ are roots of Eq. (9) and satisfy Eq. (10) for the $i$ th layer. After some algebraical manipulation, we obtain the matrizant of the $i$ th layer as

$$
\mathbf{E}^{(i)}\left(h_{i}\right)=\alpha^{(i)}\left(\begin{array}{cc}
\mathbf{E}_{1}^{(i)} & \frac{1}{k c_{66}^{(0)}} \mathbf{E}_{2}^{(i)} \\
k c_{66}^{(0)} \mathbf{E}_{3}^{(i)} & \mathbf{E}_{4}^{(i)}
\end{array}\right)
$$

where

$$
\alpha^{(i)}=\left\{\begin{array}{lll}
\frac{1}{\sqrt{P^{(i)}-\left(S^{(i)}\right)^{2} / 4},} & \text { if } & \left(S^{(i)}\right)^{2}-4 P^{(i)}<0 \\
\frac{1}{\sqrt{\left(S^{(i)}\right)^{2}-4 P^{(i)}},} & \text { if } & \left(S^{(i)}\right)^{2}-4 P^{(i)}>0
\end{array}\right.
$$


The matrices $\mathbf{E}_{j}^{(i)}(j=\overline{1,4})$ are two-by-two matrices whose entries are dimensionless and equal

$$
\begin{aligned}
& E_{1}^{(i)}(1,1)=m_{i}+p_{i} a_{i}, \\
& E_{1}^{(i)}(1,2)=\left[n_{i} e_{3}^{(i)}+q_{i}\left(b_{i} e_{3}^{(i)}-d_{i}\right)\right] e_{2}^{(i)}, \\
& E_{1}^{(i)}(2,1)=-n_{i}+q_{i}\left(d_{i}-a_{i}\right), \\
& E_{1}^{(i)}(2,2)=m_{i}+q_{i} b_{i}, \\
& E_{2}^{(i)}(1,1)=\frac{\left[n_{i}+q_{i}\left(a_{i}-c_{i} e_{3}^{(i)}\right)\right] e_{2}^{(i)}}{r_{\mu}^{(i)}}, \\
& E_{2}^{(i)}(1,2)=\frac{p_{i} c_{i}}{r_{\mu}^{(i)}}, \\
& E_{2}^{(i)}(2,1)=-E_{2}^{(i)}(1,2), \\
& E_{2}^{(i)}(2,2)=\frac{n_{i}+q_{i}\left(b_{i}-c_{i}\right)}{r_{\mu}^{(i)}} \\
& E_{3}^{(i)}(1,1)=\left[-n_{i} x^{(i)}+q_{i}\left(d_{i}-a_{i} x^{(i)}\right)\right] r_{\mu}^{(i)}, \\
& E_{3}^{(i)}(1,2)=-p_{i} d_{i} r_{\mu}^{(i)}, \\
& E_{3}^{(i)}(2,1)=-E_{3}^{(i)}(1,2), \\
& E_{3}^{(i)}(2,2)=\left[\left(n_{i}+q_{i} b_{i}\right)\left(e_{1}^{(i)}-e_{2}^{(i)}\left(e_{3}^{(i)}\right)^{2}-x^{(i)}\right)+e_{2}^{(i)} e_{3}^{(i)} q_{i} d_{i}\right] r_{\mu}^{(i)}, \\
& E_{4}^{(i)}(1,1)=E_{1}^{(i)}(1,1), \\
& E_{4}^{(i)}(1,2)=-E_{1}^{(i)}(2,1), \\
& E_{4}^{(i)}(2,1)=-E_{1}^{(i)}(1,2), \\
& E_{4}^{(i)}(2,2)=E_{1}^{(i)}(2,2),
\end{aligned}
$$

where

$$
r_{\nu}^{(i)}=\frac{c_{66}^{(0)}}{c_{66}^{(i)}} \frac{\rho^{(i)}}{\rho^{(0)}}, \quad r_{\mu}^{(i)}=\frac{c_{66}^{(i)}}{c_{66}^{(0)}},
$$

and

$$
\begin{aligned}
& a_{i}=-e_{2}^{(i)}\left(e_{3}^{(i)}+x^{(i)}\right), \quad b_{i}=e_{1}^{(i)}-x^{(i)}-e_{2}^{(i)} e_{3}^{(i)}\left(e_{3}^{(i)}+1\right), \\
& c_{i}=e_{2}^{(i)}\left(e_{3}^{(i)}+1\right), \quad d_{i}=x^{(i)}-e_{1}^{(i)}-e_{3}^{(i)} a_{i},
\end{aligned}
$$

and $m_{i}, n_{i}, p_{i}, q_{i}$ are defined as the follows. 
(1) If $\left(S^{(i)}\right)^{2}-4 P^{(i)}<0$,

$$
\begin{aligned}
m_{i}= & -\sinh \left(\varepsilon \bar{h}_{i} f_{i}\right) \sin \left(\varepsilon \bar{h}_{i} g_{i}\right) F_{i}+\cosh \left(\varepsilon \bar{h}_{i} f_{i}\right) \cos \left(\varepsilon \bar{h}_{i} g_{i}\right) G_{i}, \\
n_{i}= & \frac{1}{\sqrt{P_{i}}}\left[\sinh \left(\varepsilon \bar{h}_{i} f_{i}\right) \cos \left(\varepsilon \bar{h}_{i} g_{i}\right)\left(f_{i} G_{i}+g_{i} F_{i}\right)+\right. \\
& \left.-\cosh \left(\varepsilon \bar{h}_{i} f_{i}\right) \sin \left(\varepsilon \bar{h}_{i} g_{i}\right)\left(f_{i} F_{i}-g_{i} G_{i}\right)\right], \\
p_{i}= & \sinh \left(\varepsilon \bar{h}_{i} f_{i}\right) \sin \left(\varepsilon \bar{h}_{i} g_{i}\right), \\
q_{i}= & \frac{1}{\sqrt{P_{i}}}\left[\cosh \left(\varepsilon \bar{h}_{i} f_{i}\right) \sin \left(\varepsilon \bar{h}_{i} g_{i}\right) f_{i}-\sinh \left(\varepsilon \bar{h}_{i} f_{i}\right) \cos \left(\varepsilon \bar{h}_{i} g_{i}\right) g_{i}\right]
\end{aligned}
$$

where

$$
\begin{aligned}
& F_{i}=\frac{S^{(i)}}{2}, \quad G_{i}=\sqrt{P^{(i)}-F_{i}^{2}}, \\
& f_{i}=\frac{1}{\sqrt{2}} \sqrt{\sqrt{F_{i}^{2}+G_{i}^{2}}+F_{i}}, \quad g_{i}=\frac{1}{\sqrt{2}} \sqrt{\sqrt{F_{i}^{2}+G_{i}^{2}}-F_{i}}
\end{aligned}
$$

and $\epsilon=k h, \bar{h}_{i}=h_{i} / H(i=\overline{1, n})$.

(2) If $\left(S^{(i)}\right)^{2}-4 P^{(i)}>0$

$$
\begin{aligned}
m_{i} & =\cosh \left(\varepsilon \bar{h}_{i} b_{3}^{(i)}\right)\left(b_{1}^{(i)}\right)^{2}-\cosh \left(\varepsilon \bar{h}_{i} b_{1}^{(i)}\right)\left(b_{3}^{(i)}\right)^{2}, \\
n_{i} & =\frac{\sinh \left(\varepsilon \bar{h}_{i} b_{3}^{(i)}\right)}{b_{3}^{(i)}}\left(b_{1}^{(i)}\right)^{2}-\frac{\sinh \left(\varepsilon \bar{h}_{i} b_{1}^{(i)}\right)}{b_{1}^{(i)}}\left(b_{3}^{(i)}\right)^{2}, \\
p_{i} & =\cosh \left(\varepsilon \bar{h}_{i} b_{1}^{(i)}\right)-\cosh \left(\varepsilon \bar{h}_{i} b_{3}^{(i)}\right) \\
q_{i} & =\frac{\sinh \left(\varepsilon \bar{h}_{i} b_{1}^{(i)}\right)}{b_{1}^{(i)}}-\frac{\sinh \left(\varepsilon \bar{h}_{i} b_{3}^{(i)}\right)}{b_{3}^{(i)}}
\end{aligned}
$$

where

$$
b_{1}^{(i)}=\sqrt{\frac{S^{(i)}+\sqrt{\left(S^{(i)}\right)^{2}-4 P^{(i)}}}{2}}, \quad b_{3}^{(i)}=\sqrt{\frac{S^{(i)}-\sqrt{\left(S^{(i)}\right)^{2}-4 P^{(i)}}}{2}} .
$$

Note that in both cases of $\left(S^{(i)}\right)^{2}-4 P^{(i)}$ positive and negative, quantities $m_{i}, n_{i}, p_{i}, q_{i}$ are always real that makes matrices $\mathbf{E}_{j}^{(i)}(j=\overline{1,4})$ real. And due to the special form of matrices $\mathbf{E}^{(i)}$ (in Eq. (36)), their product denoted by $\mathbf{E}$ in Eq. (26) has the same form. This could be proved easily by direct matrix multiplication. In particular,

$$
\mathbf{E}(H)=\left(\prod_{i=1}^{n} \alpha^{(i)}\right)\left(\begin{array}{cc}
\mathbf{E}_{1} & \frac{1}{k c_{66}^{(0)}} \mathbf{E}_{2} \\
k c_{66}^{(0)} \mathbf{E}_{3} & \mathbf{E}_{4}
\end{array}\right)
$$


where

$$
\overline{\mathbf{E}}(H):=\left(\begin{array}{ll}
\mathbf{E}_{1} & \mathbf{E}_{2} \\
\mathbf{E}_{3} & \mathbf{E}_{4}
\end{array}\right)=\prod_{i=1}^{n}\left(\begin{array}{ll}
\mathbf{E}_{1}^{(i)} & \mathbf{E}_{2}^{(i)} \\
\mathbf{E}_{3}^{(i)} & \mathbf{E}_{4}^{(i)}
\end{array}\right) .
$$

Note that this matrix is dimensionless and real and it will be used to find the secular equation instead of the matrizant $\mathbf{E}(H)$ which has dimension and could be complex due to the factors $\left(\prod_{i=1}^{n} \alpha^{(i)}\right)$ and $\left(k c_{66}^{(0)}\right)$ in Eq. (45).

Inspecting Eq. (31) and Eq. (45), we see that

$$
\mathbf{M}=\left(\prod_{i=1}^{n} \alpha^{(i)}\right)^{2} k c_{66}^{(0)}\left[k c_{66}^{(0)} \bar{M}_{1}, \bar{M}_{2}, \bar{M}_{3}, \bar{M}_{4}, \bar{M}_{5}, \frac{\bar{M}_{6}}{k c_{66}^{(0)}}\right],
$$

where the dimensionless vector $\overline{\mathbf{M}}=\left[\bar{M}_{1}, \bar{M}_{2}, \bar{M}_{3}, \bar{M}_{4}, \bar{M}_{5}, \bar{M}_{6}\right]^{T}$ is defined similarly to vector $\mathbf{M}$ in Eq. (31) in which the matrix $\mathbf{E}$ is replaced by the dimensionless matrix $\overline{\mathbf{E}}$.

Substituting vectors $\mathbf{M}$ and $\mathbf{V}$ from Eq. (47) and Eq. (32) into the secular equation, Eq. (30), it can be written as

$$
\left(b_{1}^{(0)}-b_{2}^{(0)}\right) k^{2}\left(c_{66}^{(0)}\right)^{4}\left(\prod_{i=1}^{n} \alpha^{(i)}\right)^{2} \overline{\mathbf{M}} \overline{\mathbf{V}}=0 .
$$

Since $b_{1}^{(0)}-b_{2}^{(0)} \neq 0$ and $\alpha^{(i)} \neq 0$, this equation could be expressed as

$$
\overline{\mathbf{M}} \overline{\mathbf{V}}=0 .
$$

Note that this is a real equation of dimensionless frequency $\epsilon$ and velocity $x^{(0)}$, and dimensionless material parameters of layers and half-space.

\section{NUMERICAL CALCULATION}

For a given set of material parameters of layers and half-space, all the dimensionless parameters are computed by Eqs. (34), (39). Our aim is to compute the phase velocity of Rayleigh waves in the form $x^{(0)}$ for a given wave number expressed in form of $\epsilon$. First, we compute vector $\overline{\mathbf{V}}$ in the secular equation, Eq. (49), by Eqs. (33). To calculate vector $\overline{\mathbf{M}}$, we compute all the matrizant of layers in dimensionless form by Eq. (26), then use Eq. (46) to compute their product to obtain the matrix $\overline{\mathbf{E}}$. The vector $\overline{\mathbf{M}}$ is then computed by Eq. (31) in which the matrix $\mathbf{E}$ is replaced by $\overline{\mathbf{E}}$. Finally, the phase velocity $x^{(0)}$ is found by setting the scalar product of vectors $\overline{\mathbf{V}}$ and $\overline{\mathbf{M}}$ equal zero.

Table 1. Properties of Materials

\begin{tabular}{|c|c|c|c|c|c|}
\hline Material & $\mathbf{E}_{\mathbf{1}}(\mathbf{G P a})$ & $\mathbf{E}_{\mathbf{2}}(\mathbf{G P a})$ & $\mathbf{G}_{\mathbf{1 2}}(\mathbf{G P a})$ & $\boldsymbol{\nu}_{\mathbf{1 2}}$ & $\boldsymbol{\rho}\left(\mathbf{g} / \mathbf{c m}^{\mathbf{2}}\right)$ \\
\hline Graphite/epoxy & 30.00 & 0.750 & 0.375 & 0.2500 & 1.9 \\
\hline Carbon/epoxy & 142.17 & 9.255 & 4.795 & 0.334 & 1.9 \\
\hline Glass/epoxy & 38.49 & 9.367 & 3.414 & 0.2912 & 2.66 \\
\hline Steel & 77.4 & 77.4 & 29.025 & 0.3333 & 7.9 \\
\hline
\end{tabular}




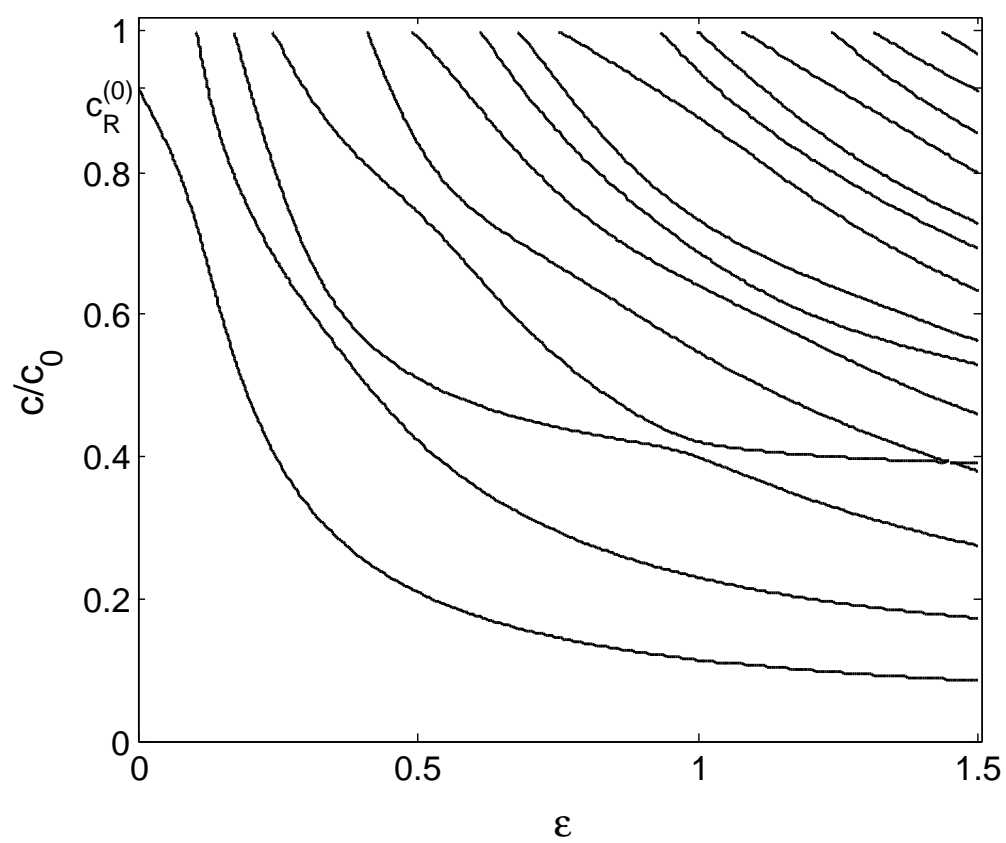

Fig. 2. Phase velocity dispersion of some first modes for orthotropic layered half-space given in Tab. 1.

To illustrate the method, we consider a model of orthotropic layered half-space consisting of three layers overlying a half-space with material properties given in Tab. 1 (see Liu and Xi, 2002, Chapter 3 [15]). All layers are assumed to have the same thickness. The dispersion curve of this model is shown in Fig. 2 with several first modes. The $x$-axis is the dimensionless frequency $\epsilon=k H$ where $H$ is the sum of thicknesses of layers. The $y$-axis is the ratio of the phase velocity to the shear wave velocity of the half-space in the principal direction $x_{1}$-axis denoted by $c_{0}=\sqrt{c_{66}^{(0)} / \rho^{(0)}}$. It can be seen from the figure that the threshold velocity of higher modes is $c_{0}$ as the result of condition given in (35).

Note that, when frequency gets smaller, the phase velocity approaches to the Rayleigh velocity of the half-space denoted by $c_{R}^{(0)}$. For the given material parameters of the half-space, this velocity could be computed by a formula given in Vinh and Ogden [14] as

$$
\bar{c}_{R}^{(0)}=\frac{c_{R}^{(0)}}{c_{0}}=0.9194 .
$$

This result is consistent with the our numerical calculation.

\section{CONCLUSION}

By using the Sylvester's theorem, the matrizant of a layer is expressed explicitly. Its equivalent form for the problem of finding dispersive curve of Rayleigh surface waves is then obtained. The equivalent form of the matrizant is always real and expressed in terms 
of the dimensionless variables and parameters. The problem of the vertical wave number in the half-space being complex due to the anisotropy is handled by using the Vieta's formulas for the propagation equation to obtain the secular equation of Rayleigh waves in convenient form to numerical calculation. A numerical illustration for the dispersive curves of Rayleigh waves in an orthotropic layered half-space is presented.

\section{ACKNOWLEDGMENT}

The work was supported by the Vietnam National Foundation for Science and Technology Development (NAFOSTED) under grant 107.02-2014.17.

\section{REFERENCES}

[1] W. T. Thomson. Transmission of elastic waves through a stratified solid medium. Journal of Applied Physics, 21, (2), (1950), pp. 89-93.

[2] N. A. Haskell. The dispersion of surface waves on multilayered media. Bulletin of the Seismological Society of America, 43, (1), (1953), pp. 17-34.

[3] L. Knopoff. A matrix method for elastic wave problems. Bulletin of the Seismological Society of America, 54, (1), (1964), pp. 431-438.

[4] J. W. Dunkin. Computation of modal solutions in layered, elastic media at high frequencies. Bulletin of the Seismological Society of America, 55, (2), (1965), pp. 335-358.

[5] B. L. Kennett. Seismic wave propagation in stratified media. Cambridge University Press, (1983).

[6] X. Chen. A systematic and efficient method of computing normal modes for multilayered half-space. Geophysical Journal International, 115, (2), (1993), pp. 391-409.

[7] S. Crampin. The dispersion of surface waves in multilayered anisotropic media. Geophysical Journal International, 21, (3), (1970), pp. 387-402.

[8] S. Crampin and D. B. Taylor. The propagation of surface waves in anisotropic media. Geophysical Journal International, 25, (1-3), (1971), pp. 71-87.

[9] F. I. Solyanik. Transmission of plane-waves through a layered medium of anisotropic materials. Soviet Physics Acoustics-USSR, 23, (6), (1977), pp. 533-536.

[10] S. I. Rokhlin and Y. J. Wang. Equivalent boundary conditions for thin orthotropic layer between two solids: Reflection, refraction, and interface waves. The Journal of the Acoustical Society of America, 91, (4), (1992), pp. 1875-1887.

[11] T. C. T. Ting and C. O. Horgan. Anisotropic elasticity: theory and applications, Vol. 405. Oxford University Press New York, (1996).

[12] R. A. Frazer, W. J. Duncan, and A. R. Collar. Elementary matrices and some applications to dynamics and differential equations. Cambridge University Press, (1938).

[13] H. Takeuchi and M. Saito. Seismic surface waves. Methods in Computational Physics, 11, (1972), pp. 217-295.

[14] P. C. Vinh and R. W. Ogden. Formulas for the rayleigh wave speed in orthotropic elastic solids. Archives of Mechanics, 56, (3), (2004), pp. 247-265.

[15] G. R. Liu and Z. C. Xi. Elastic waves in anisotropic laminates. CRC press, (2002). 